\title{
Gibbs' rational reconstruction of thermodynamics according to the heuristic tradition of Descartes' analytical method
}

\author{
Jojomar Lucena $^{1}$, Cássio Costa Laranjeiras ${ }^{* 2}$, José Raimundo Novaes Chiappin ${ }^{3}$ \\ ${ }^{1}$ Universidade de São Paulo, Departamento de Filosofia, São Paulo, SP, Brasil \\ ${ }^{2}$ Universidade de Brasília, Instituto de Física, Brasília, DF, Brasil \\ ${ }^{3}$ Universidade de São Paulo, Departamento de Economia, São Paulo, SP, Brasil
}

Received on January 16, 2018; Revised on May 30, 2018; Accepted on June 12, 2018.

\begin{abstract}
Many rational aspects of some fields of physics, including those related to the process of discovery, may be better understood using methodological tools typical of the philosophical analysis of science. Throughout this paper we shall use the heuristic tradition of Descartes' analytical (or discovery) method for a rational reconstruction of Gibbs' thermodynamics, or thermodynamics of potentials, seeking to emphasize the potentialities of the representational character of science. Our contention is that Descartes's method of discovery illuminates the intellectual itinerary of the construction of the thermodynamics of potentials.
\end{abstract}

Keywords: Gibbs, Descartes, Analytical Method, Thermodynamics of Potentials, Heuristics of Representation.

\section{Introduction}

In general, we can say that the goal of science is to produce adequate and satisfactory explanations for phenomena that strike us. The "adequate" and "satisfactory" qualifications refer to those explanations that obey testable and falsifiable universal laws, constructed from a given set of initial conditions. However, the phenomena to be explained do not always correspond to something never observed before - for example, the discovery of the magnetic effects generated by electric currents (1823), which brought about an authentic "scientific race." Scientific curiosity may focus on already established experimental laws, seeking universal rules from which the former could be inferred; Even universal laws or theories can become the target of the theoretical scientist, in search of principles or theories with ever richer content and a higher degree of universality [1, pp.190-192]. It is in this context that we defend the idea that Gibbs's work - by reformulating Clausius's thermodynamics as the thermodynamics of potentials - expresses this quest.

*Correspondence email address: cassio@unb.br
To carry out this work, Gibbs used skills that involve a reflection on scientific activity itself - and, to a certain extent, belongs more to the field of the philosophy of science or epistemology. The demarcation line between science and the philosophy of science is far from clear and indisputable, but the reflection on scientific activity, although equally performed by scientists, is the prime object of the philosophy of science or epistemology. Thus, the reformulation of thermodynamics developed by Gibbs constitutes an excellent example of a theory elaborated according to a heuristic tradition that we identify with Descartes' method of analysis, to which he also refers as a method of construction or discovery.

From this perspective, discovery becomes amenable to rational reconstruction, since a theoretical construction like Gibbs, carried out according to the method of analysis, is also a discovery. However, this perception was contradicted or ignored by the prevailing philosophy of science during the first half of the $20^{t h}$ century, logical positivism. In fact, the interest in the thermodynamics of potentials transcends the frontiers of physics, also becoming an object of study in epistemology. To explore this issue, which we believe to be valuable to scientists 
and not just philosophers, it is necessary to introduce a series of concepts proper to epistemology. This will be the goal of section 2 . In section 3 , the heuristic tradition of Descartes's analytical method will be presented, seeking to provide a framework for the discussion of Gibbs's thermodynamics, which will be the subject of section 4 .

\section{The context of discovery in science}

One of the leading philosophers of science in the $20^{\text {th }}$ century and one of the most fervent advocates of logical positivism (also known as neopositivism or logical empiricism) was Hans Reichenbach (1891-1953), for whom rational reconstruction - the object under consideration in epistemology - corresponds to a "form in which thinking processes are communicated to others persons instead of the form in which they are subjectively performed" 2 , p.6]. As such, he established that epistemology is interested in logic and not the psychology of these processes. Following this demarcation of terrains, he introduces the distinction between the context of discovery and the context of justification - just a few years after the publication of Popper's "The Logic of Scientific Discovery" [3]-, placing discoveries (in science) as the result of merely psychological processes and, therefore, outside the scope of interest of logic [2, p.30]. This corresponds to the claim that science, especially physics, does not discuss how the author came to a specific solution, but how he justifies it. In the case of Newtonian mechanics, this would be to say that epistemology does not investigate how the author conceived his expression of force, but how, once incorporated into the system, that vector quantity resulted in the particular laws of motion that were experimentally verified. Many articles - especially by classic authors, Gibbs among them - explores this process.

In addition to Reichenbach and Popper, Hempel [4] (implicitly), Feigh [5], Salmon [6] and others shared this view about the scope and limits of this distinction, which is a reflection of the understanding of philosophy as the philosophy of language, whose normative evaluation of propositions, that is, its justification, should be carried out logically, leaving aside other criteria, such as the methodological criterion. Specifically on this topic, Hoyningen-Huene identifies five versions of the distinction between the context of discovery and the context of justification, of which the third is the fact that discovery is described empirically, and justification, logically [7]. According to the reductionism of logical positivism, therefore, discovery is very often described according to its insights through images or analogies that reproduce the psychological states or processes of the scientist's mind, as in the paradigmatic discovery of benzene's ring structure by Kekulé [8].

The logical demarcation of the justification context is not characteristic nor exclusive to the logical empiricism of the $20^{t h}$ century. Even before, many authors shared the view that there is neither logic nor method to discovery.
This is the case, for example, of the British philosopher and science historian William Whewell. A follower of Hume and a contemporary of the great discoveries in the field of thermodynamics and electromagnetism in the $19^{\text {th }}$ century, he saw that the deductive method could not be separated from the inductive method because "deduction is a necessary part of induction. Deduction justifies by calculation what induction had happily guessed" 9 , p. 115]. For him, discovery was not merely the product of the combination of particular facts, but of their union through the act of thinking, to formulate a more general concept, that combines them [9, p.72]. This act of thinking, however, could not be ordered according to a method. The general concept supplied by the mind is invented and can be associated with other facts or concepts on the same level, from which more general concepts or principles are induced, like climbing a ladder in which the ascension to the next rung is done through a movement of induction [9, p.114]. The inductive movement is not justified in and of itself, but in the deductive demonstration, which shows, as in a downward movement, the most general principles of particular facts. If invention cannot be justified in this way, it cannot be called an invention. It is in this sense that deduction is a necessary part of induction.

Although Whewell presents the scientific method as being predominantly an inductive method - it should start with data provided by rigorous scientific experimentation, it is justified, in the strictest sense, only through its complementary deductive movement. Discovery remains, as it were, outside the space of demonstration, of logic, pushed aside as a psychological phenomenon. Furthermore, the logical entity of induction is insufficient, a fact which, given the relationship between induction and discovery, casts this last concept even further away from the terrain of justification.

From this angle, two paths can be followed to try to reintroduce discovery, albeit only partially, in the terrain of justification. In the first path, it is necessary to recognize a certain independence of induction in relation to deduction as a method of demonstration to remove discovery from the labyrinth of psychology, which amounts to giving it certain powers of evidence and logic. This path, however, is strenuous and the result is not free of problems, as testified by Carnap's work on probability and inductive logic. The second path is not new and will be revisited here. In the $17^{\text {th }}$ century, Descartes rigorously formulated it, disconnecting induction and discovery, the latter being understood by him as the result of a method: analysis.

\section{Descartes' method of discovery}

There is a chronological distance of more than two centuries between Descartes and Gibbs. Even so, their methods to approach scientific problems bring them extremely close to each other. Without fear of exaggeration, Descartes 
can be identified as the main thinker behind the new image of the modern world, both political and scientific. The substantial dualism between the cogito and the masses in motion is the pillar of these two worlds (the socio-political and the natural). Descartes ushered in modernity not only ontologically, but also epistemologically and methodologically $[10$. Taking geometry as a model for the organization of knowledge, Descartes created the transcendental problem with the postulation that, from knowledge to being, the consequence is good. Thus, he stated that our knowledge of the outside world is always an intellectual or theoretical knowledge, which uses ideas and concepts to represent the outside world and to uphold the pretense of verisimilitude. Knowledge must therefore be submitted to empirical testing after its internal consistency has been assessed.

As if this were not enough, the adoption of geometry as the model for the organization of knowledge has a boundless heuristic potential. At the beginning of $L a$ Géométrie [11], Descartes introduces a reference system in accordance with which a unity is established to teach how to add, subtract, divide, multiply and take the square root of segments of straight lines, whose results are once again segments of straight lines, and not rectangles or solids, as was traditionally done (in the case of multiplication), and therefore limited to three dimensions. This stratagem allowed him to expand the space from three to $n$ dimensions, and so he transformed the Euclidean geometric space, which was supposed to represent physical space, into a purely intellectual space of $n$ dimensions. With this simple idea, he created a reference system with unity, built on the intersections of the straight lines of Thales's theorem, which is a theorem of proportions of parallel straight lines cut by transversal straight lines, for any angle. In other words, it is a system of rules to operate with segments and produce segments, converting geometric problems into algebraic problems and vice versa.

Here we have the origin of one of the most powerful tools to create ideas and solve scientific problems: the representation of a problem can be made in different languages, maintaining the same informational content; and the adoption of a specific representation can significantly simplify the solution to a problem. With Descartes's reference system and his rules for the conversion of geometric figures into algebraic equations, the same problem can be represented in different ways depending on the adopted reference system, and it is possible to ensure the transition from one system to the other through rules without any loss of informational content. Even more relevantly, the solution to a problem may be simpler in a given representation than in another. Effectively, an essential part of solving a problem is to represent it adequately.

The first problem solved by Descartes in Geometry, after establishing his method of constructing algebraic equations of geometric problems, is attributed to Pappus, the so-called Pappus's four line loci problem. In fact, this problem had been elaborated by Euclid and taken up by Apollonius, but neither one nor the other resolved it satisfactorily. Pappus then provides a solution for the simplest cases, these of four and five lines. For larger numbers of lines, the problem remains either by the intrinsic complexity or by the constraint imposed by the purely geometric conception of the problem involving, in its formulation, the comparison between figures: rectangle with square (four line loci problem), rectangle with rectangle (five line loci problem) and parallelepiped with parallelepiped (six line loci problem). The comparison is made employing the areas or volumes of the figures - and always area with area and volume with volume -, which correspond, respectively, to products of two or three straight lines [11, pp. 304-311]. For products with more terms, the old geometry was not able to attribute a meaning ${ }^{1}$

By using algebraic representation, Descartes not only determines the solution curves for the original problems but extends the comparison for products with more than three straight lines. In the movement toward solution, the first step is to consider the problem solved, naming all the lines that seem necessary for the construction, also those that are unknown. In particular, there are two segments formed by the straight lines and extensions that Descartes calls $\mathrm{x}$ and $\mathrm{y}$. Since the angles between the lines are given, they can all be expressed as a function of $\mathrm{x}$ and $y$. The second step is to take expressions to the same straight line and equate them, assembling equations. The idea is to continue with this procedure until you find an equation where the unknown data is just $\mathrm{x}$ and $\mathrm{y}$. This infinite amount of points form a locus, which can and must be traced, according to Descartes.

This final equation is the solution of the problem in algebraic language, incomprehensible, therefore, to the old geometers. To make it understandable, it must be translated in terms of straight lines, conic sections, and other curves that can be accurately drawn (certainty). The end result can be summarized as follows:

When the number of lines does not exceed five, and the lines do not intersect at right angles, he argues that the locus will be described by a quadratic equation and can thus be found using a ruler and a compass. When the number of lines is between five (intersecting at right angles) and nine lines, the equation becomes either a cubic or a quartic equation and can thus be found using conic sections. For problems with nine lines intersecting at right angles, or up to thirteen lines intersecting at non-right angles, the answer is an equation of the fifth or sixth degree which

\footnotetext{
${ }^{1}$ We purposely avoided describing Pappus's problem because, although it involves only straight lines, clear enunciation of it requires a series of details, which certainly needs much more space.
} 
can be constructed with curves more complex than the conic sections [12, p.8].

Descartes's method of translating to algebra allows him to solve and generalize geometric problems at hand. It is a new and powerful way of thinking about the old geometrical problems. However, due to this novelty, the algebraic equation solution of the problem had to be expressed entirely in geometric terms, the mathematical language known at that time together with the theory of proportions, which restricted the legitimacy of the method to the curves that could be traced precisely to the resources of the time $[12$, p. 24]. As this requirement was loosened, a fact related to the very development of algebra and the birth of Calculus, the program put forward by Descartes in La Géométrie, to combine geometry and algebra to solve any geometrical problem, became a reality. With these ideas, Descartes changed two thousand years of history.

It is equally interesting to realize that this is the one thing that makes Gibbs replace the representation by cycles with the representation by potentials in thermodynamics, employing the resources of the diagrammatic and geometric representation to finally arrive at the formulation of algebraic potentials. Going further, we can conclude that the construction of the representation of thermodynamics, from cycles to potentials, is a process that simulates the analytical method defended by Descartes as the method of discovery which in fact consists in a method to construct representations $[13$.

It is worthwhile, for the moment, to make some considerations about Cartesian analysis. Even though Descartes applied this method in his scientific works, such as $L a$ Géométrie, La Dioptrique and Les Météores (which follow the Discours de la Méthode, which served as an introduction to them), it can be argued that Descartes does not present analysis and synthesis as parts of the scientific method anywhere as clearly and succinctly as in the response to the second objection in Les Méditations Métaphysiques. On the other hand, Whewell removed the issue of discovery from the context of induction and deduction and introduced it in the context of analysis and synthesis. For Descartes, both analysis and synthesis possess the quality of justification, which in Whewell lies only in the deductive movement. Through them it is possible to demonstrate, but in a different way. The first one uses invention, while the second one uses composition (construction) or exposure [14, p.177]. However, order characterizes both modes of demonstration. It is a logical order, since the propositions that come after rely solely on those that come before, and those that come before cannot depend on anything that comes after. It is in this sense that analysis and synthesis are structured methods according to a logical order.

There is a disparity between the Latin original and the French translation authorized by Descartes, and made by Clerselier, which has been detected and analyzed by several reviewers. This is not the proper place to dwell on this interesting question, but it is necessary to understand both texts harmonically and, to this end, we choose to follow Loparic's interpretation 15] as the most convincing one. With this in mind, the original text says that "analysis shows the true way by which a thing was methodically discovered and derived, as if a priori" - here the conjunction "as if" suggests that analysis is not a priori, but simply simulates an a priori procedure, and the French text adds that discovery comes to be through a procedure that goes from the effects to the causes, assuming the solution to the problem is a given. In mathematics, this consists in assuming the unknown factor as given or the theorem to be proven as true. This clarification concerning the term a priori is essential, since for medieval thinkers an argument is a priori if it goes from cause to effect, from principle to consequence, while an argument is a posteriori if it connects the effects to the causes, the consequences to the principles, in apparent disagreement with the Cartesian texts.

The Latin text then mentions that "synthesis, on the other hand, works in the opposite direction, retracing the path, so to speak, a posteriori (although the proof itself is, in this case, often more a priori than in the other), clearly demonstrating what is contained in its conclusions", here the adverb a posteriori indicates that the synthesis starts with the conclusion reached by the analysis, while the a priori in parentheses indicates that the proof in the synthesis occurs through a process that goes from cause to effect, as the French translation makes clear Loparic explains "the typical usage of synthesis is not to 'examine' what follows from the causes, but to start with known causes to prove (a priori) the existence of this or that effect" 15, pp.148-149].

As methods, therefore, analysis and synthesis have different attributes, which allow the first to be characterized as a procedure of discovery and the second as an exposition of already acquired truths. Analysis precedes synthesis, although the latter may be presented separately, outside the context of discovery, as in Euclidean geometry. This possibility of isolating synthesis has nothing to do with a supposed need for analysis to logically complement synthesis, as we can see occurring between induction and deduction in Whewell. In Descartes, analysis and synthesis are independent ways to demonstrate, a relation that was not so explicit even in the Discourse on the Method, as emphasized by Battisti:

So far, following the example of the Greek geometricians, the Cartesian method was generally composed of two complementary stages, even if sometimes linked together or even presented separately (especially in mathematics). In this case, analysis presents itself as selfsufficient even when demonstrative. However, this characteristic is not absolutely new or unexpected. First, because this phenomenon occurred within the history of mathematics, to the extent that synthesis became indepen- 
dent from analysis, albeit not as a method of discovery, but especially with the advent of algebra, through which analysis no longer needed the assurance given by synthesis (i.e., from its demonstrative part, keeping only its constructive part). Secondly, given the importance attributed to discovery, Descartes has always considered the analytical step as critical, reserving the reorganization of the steps or the exposition of the problem's solution for synthesis, along with the proof of some of the relationships that made it possible. [16, p.350]

The decomposition of the scientific method into two movements, the inductive and the deductive, as Whewell prefers to put it, where the first one is only demonstrated in the second, is not juxtaposed to Descartes's conception in Les Méditations. The reason for this is that analysis is not directly associated to induction, not even for Whewell, for whom "geometric deduction (and deduction in general)is called synthesis, because we introduce, at successive steps, the results of the new principles. But in reasoning on the relations of space, we sometimes go on separating truths in their component truths, and these in other component truths, and so on. And this is geometric analysis" [9. p.12]. Here, synthesis and deduction are synonymous, both for Whewell and Descartes, but Whewell's analysis does not involve the upward movement inherent to induction, while deduction descends on the same ladder, whose top was established through induction in a single leap [9, p.114]. It is, in a single term, inventive, but not demonstrative, and therefore it remains limited to the scope of psychology, outside the space of the method.

According to Whewell's categories, Cartesian analysis is a combination, of ascending induction, which gives it an a priori character, and classical analysis performed a posteriori, inferring the cause from the effects. The incorporation of induction gives it an inventive character, while the classical analysis gives it a methodological character and, as such, a certain justification power, which should not be understood as inferior in comparison with deduction, but as different, of another nature.

Descartes is far from to be original in the characterization and use of methods of analysis and synthesis. In fact, both methods refer back to classical antiquity. Hintikka, in a celebrated work, reconstructs the methods from the "Collections" book of Pappus and in the light of other classic works of antiquity. To obtain a similarity to Descartes descriptions, it is enough to observe how Hintikka summarized the method of analysis in this context:

Analysis is a method Greek geometers used in looking for proofs of theorems and for constructions to solve problems. In both cases, analysis consists in assuming what was being sought for, in inquiring where it comes from, and in proceeding further till one reaches something already known. The analysis is followed by a synthesis in which the desired theorem or construction is established step by step in the usual manner by retracing the stages of the analysis in the reverse order [...] This concept is one of the rare avenues through which instances of non-trivial modes of logical inference have found their way into the awareness of earlier philosophers of science, logic, and mathematics" 17, pp. 1-2].

He also adds that geometrical analysis was not only one of the starting-points of Descartes' analytical geometry; it was also one of the inspirations of his general methodological ideas, as we are advocating.

The use Descartes makes of the method of analysis is extensive, covering such different subjects as epistemology, mathematics, physics, metaphysics and theology. Two examples are sufficient to evaluate the operation of this method. The first is taken from the Second Meditation, in which the philosopher tackles the problem of finding one thing that is certain and unquestionable. The search for the answer starts by recognizing, first of all, the conceptual foundation of the problem to then establish a method, the method of doubt, which enables the operationalization of the analysis and casts a doubt on all see, remember and feel; all these impressions may be fictions of the mind. As such, the very existence of the senses and the body is put in check. The question of existence, in this case, plays a central role in the method, because it represents the radicalization of doubt, faced with which the answer to the problem appears intuitively:

But I was persuaded that there was nothing in all the world, that there was no heaven, no earth, that there were no minds, nor any bodies: was I not then likewise persuaded that I did not exist? Not at all; of a surety I myself did exist since I persuaded myself of something ... - we must come to the definite conclusion that this proposition: I am, I exist, is necessarily true each time that I pronounce it, or that I mentally conceive it. [14, p.12]

Here, doubt, extended to existence itself, allows us to determine with certainty something which no doubt can affect, which is the fact of existence made evident by the act of thinking, even if wrongly. Since it could not be otherwise, the solution to the problem follows the general model of rationality formalized by Descartes, in which a foundation composed of concepts and propositions is organized by a method, understood as a system of inference with rules and criteria through which other propositions are evaluated regarding their accuracy; that is, one considers whether other propositions also belong to the foundation of the system of knowledge and, wheter this, foundation be expanded 18 
The decision whether a given proposition should belong to the foundation of a system of knowledge; consists in the solution to the proposed problem. In this sense, deciding is solving the problem. However, the system of inference with rules and criteria, as mentioned above, is not able per se to generate such a decision. In this case, the method of doubt alone does not make the proposition "I exist" clear. The system of inference must be embedded in a greater logical structure, like a routine in a computer program. One must not forget that the routine is also a program, but a program with a limited scope in relation to the logical structure. The latter is the one capable of providing a conclusive decision on a proposition, revealing whether it belongs or not to the system. The two of synthesis and analysis, as conceived by Descartes, are the logical structures to which routines should be added. The term "method", therefore, specifies both the greater algorithm for the solution to the problem (synthetic or analytic) and the algorithm that acts as a heuristic resource and operationalizes the decision method. However, for a matter of didactic, we shall avoid using the term "method" in this second sense, preferring the term "intermediate idea".

In this case, the systematic doubt take on the role of a thought experiment, in which the use of the malignant genius -which can deceive men in relation to all the impressions of the external world - promotes its radicalization. In this situation we can see that Descartes's epistemology is an epistemology with a subject, i.e, the Cartesian theory of knowledge implies (in a privileged way) a rational and conscious subject. This condition (the malignant genius) acts as the intermediate idea that allows us to connect the foundation to the first certainty of knowledge - "I am, I exist" -, thus solving the problem. It is clear that the systematic doubt will fail to provide the solution to the problem if not associated with a foundation, which can be identified or added to a hypothesis that enables the resolution. This logical order, which comprises a particular algorithm, is the one that classic rationalism often calls the method of analysis [19]. There are, therefore, two meanings the term "method" can have. The first one, more general, refers to synthetically or analytically operationalized decidability; the second one, more particular, since it focuses on the proposed problem, is the algorithm or routine, that operationalizes the analysis. Here, the method of the systematic doubt operationalizes the method of analysis with the aid of the malignant genius hypothesis.

The other example is taken from La dioptrique. In the search for an explanation of the nature of light that would also allow for a reproduction of the refraction phenomenon, Descartes uses the tennis balls model to try to understand how and why the ball deviates from an extension of the line of incidence when it passes from air to water. With this model, he understands that the deviation at the interface between the two media is the result of a change in the speed of the ball, which decreases when going from the air to the water. In comparison with the angle of incidence, therefore, the angle of refraction increases. However, what is observed in the case of Snell's Law, which describes the light refraction phenomenon, is that the angle of refraction decreases. Descartes concludes, therefore, that light is
[...] nothing else but a certain movement or an action, received in a very subtle material that fills the pores of other bodies; and you should consider that, as a ball loses much more of its agitation in falling against a soft body than against one that is hard, and as it rolls less easily on a carpet than on a totally smooth table, so the action of this subtle material can be much more impeded by the particles of air, which, being soft and badly joined, do not offer it very much resistance, than by those of water, which offer it much more; and still more by those of water than by those of glass of crystal. So that, the harder and firmer are the small particles of a transparent body, the more easily do they allow the light to pass: for this light does not have to drive any of them out of their places, as a ball must expel those of water, in order to find passage among them. 11, pp.107-108]

Given Descartes' enormous prestige, the assertion that the speed of light is greater in water and crystal than in air was challenged by few, one of them being Pierre de Fermat, who brought his solution of the problem to the public with the principle of least time, just after the death of the other 20]. Biographical details aside, Descartes represents the problem as a dynamics of shocks, with the experimental laws of collisions between hard spheres and means with different consistencies. The intermediate idea is description of the nature of the propagation of light, namely a subtle matter whose agitation and propagation is higher in more consistent (harder) means, which, when transferred to the context of the dynamics of shocks, makes possible the solution of the problem, that is, the inference of the Snell's Law. We see, therefore, that the basis of knowledge is composed of the dynamics of shocks, which Descartes himself had developed;and the model of the nature of the propagation of light in the context of dynamics of shocks is the intermediate idea that makes the method of analysis operative, given the discovery of the law of refraction 2 Schematically, in the present example, the knowledge base (shocks dynamics) plus the intermediate idea (the model of the propagation of light) constitutes the method of analysis, whose associated operation is a discovery, justified precisely by the method of analysis. This discovery is amenable to a rational reconstruction, not through deductive logic, much less an inductive process, but through the method of analysis.

\footnotetext{
${ }^{2} \mathrm{~A}$ more detailed study of the heuristics of representation in the optics of Descartes and Fermat can be found in 21].
} 
In both this and the previous example, the starting point is assumed as a given of the problem or, to use a more proper terminology for mathematics, the value of the unknown that solves the problem is assumed as given: the problem is soluble. In the second example, given the basis of knowledge, this stratagem allows the tennis ball model of light, as moving matter. The requirement for this was Cartesian metaphysics, for which the universe is reducible to extensive matter in motion. In each case, the choice of an adequate representation - with the addition of an intermediate idea - operationalizes this structure as a method, the method of analysis, that allows us to discover or to invent the solution to the proposed problem.

This is Descartes's method of discovery. As such, discovery becomes the logical outcome of a method? No, the intervention of a psychological element is still necessary. There are no recipes to determine the most appropriate representation nor the intermediate idea, which are central and indispensable parts of this inventive movement. In Descartes, what happens is that this element is reduced to its minimal expression, one in which the intuition of discovery becomes evident thanks to the construction of a representation, through the method of analysis, in which discovery becomes intuitive, without the need of other evidence but one's own intuition.

The solution, therefore, becomes part of the system of knowledge as one more given. According to the structure of the Cartesian method, however, intuition can be taken as a hypothesis and tested in accordance with its consequences, logically completing, the upward analytical movement with the downward synthetic movement. In the latter, consequences are also deducted from the hypothesis with the aid of concrete models, with other propositions being potentially obtained in addition to those that led to discovery. One movement, however, does not always follow the other in that order in time. Lagrange's works on mechanics provide a unique example of the complementarity and independence of the two movements. A little over twenty years before the publication of Mécanique Analytique 22], Lagrange had already deducted the equations of motion for a range of situations, including fluids, from the principle of least action, understood as a technique for solving problems and not as a metaphysical principle. However, it is only in Mécanique Analytique that he incorporates the principle of least action from the law of balance - which originally belongs to the context of statics - changing it to the principle of virtual velocities according to D'Alembert. The methodological movement is ascending. The empirical foundation is represented concisely in the principle of virtual velocities and, with the help of some hypotheses and differential calculus, this principle is integrated.

Inspired by the work of Lagrange, Hamilton performs a similar task in Optics. The first problem he faced was to determine the focal mirror for a system of rays, that is, to find a mirror that reflects a given bundle of rays to a single point or focus. The law of reflection, generalized to a beam in space, is represented by a linear form whose difference coefficients depend on the cosines of the angles of incidence and reflection. As the reflected rays converge to a focus, the cosines of the angles of this part are definable functions once the coordinates of the mirror are known. Thus, the problem is restricted to the incident part of the system, and the condition for the solution is that the incident beam is cut perpendicularly by a series of surfaces, with any of these being the locus of the mirror which reflects the beam to a single point. However, this condition is equivalent to saying that the linear form corresponding to the incident part is an exact differential, which can therefore be integrated, providing the analytical expression of the series of surfaces.

The sum of the distances from one of these surfaces to the mirror and from it to the focus is therefore the same for all rays of the beam, defining every surface considering a fixed mirror - as a static value for the sum of distances. After satisfying the indicated conditions, therefore, a variational principle related to the distance traveled by the light can be inferred. Hamilton notes that the subtraction between the distances traveled by the incident ray, measured from one of the perpendicular surfaces, and the reflected ray is also constant. For this reason, he gives these surfaces the name of surfaces of constant action, analogously with Mechanics, although his argument does not rely on any - corpuscular or undulatory - metaphysical statement regarding the nature of light.

Since the surfaces characterize a constant action, the associated variational principle can be equivalently expressed in terms of the representative function of the surface and no longer by the distances traveled by the rays. In this sense, instead of describing the geometric properties of the beam, the system is also characterized by the function of the series of surfaces. Hamilton calls this function a characteristic, since all the properties of the system can be deducted from it using the variational method, which translates into a set of differential equations involving the characteristic function and properties of the system. This movement, it should be noted, is descending, going from principle to empirical foundation. Subsequently, he exposes the deep affinity between the method developed for Geometric Optics and Lagrange's Mechanics, establishing the Lagrangian and Hamiltonian functions as characteristic functions in Mechanics 3

\section{Gibbs' thermodynamics}

In physics, there are few and rarely intentional examples of the application of Descartes' method of analysis. One

\footnotetext{
${ }^{3}$ This is a summary of the first six sections of "Theory of systems of rays" (Transactions of the Royal Irish Academy, v. 15, 1828, p. 69-174), where Hamilton applies his method to reflection optics. For Refraction and the relationship with Analytical Mechanics, see 23 .
} 
of these, however, is the construction of Gibbs' thermodynamics, also known as the thermodynamic of potentials. Before Gibbs, the science of heat could be called the thermodynamics of cycles, because in it Carnot's cycle - which reproduces the operation of a reversible (ideal) machine - reveals itself both as the heuristic instrument enabling the formulation of the law of conservation of energy and the concept of entropy, and as a model from which the relations between thermodynamic quantities are deducted in the form of empirically testable laws ${ }^{4}$. As an indispensable resource for the thermodynamic of cycles, which simplifies the first law to $d^{\prime} Q=d^{\prime} W^{5}$ (where $\mathrm{Q}$ indicates the heat exchanged and $\mathrm{W}$ the mechanical work performed), the operation of thermal machines and thermodynamic phenomena are represented in pressure by volume diagrams $(\mathrm{PxV})$, which make it possible to extract the amount of work by means of the geometric properties of the area circumscribed by the cycle. This is how Clapeyron proceeded to deduce the equation that bears his name and which relates the pressure variation to the temperature variation for substances in state changes, as did Maxwell to establish his homonymous relations.

Further details about the characterization of classical thermodynamics - historically from Carnot to Clausius, including part of Maxwell's research - as the thermodynamics of cycles are in reference 25]. For the present study, it is enough to know that the qualification "of cycles" corresponds to the fact that the diagrammatic representation is the central resource for the investigation and solution of problems in this historical stage of science of the heat. Hence, the thermodynamics of cycles can also be called diagrammatic thermodynamics, because the empirical basis is often represented in this way, and the properties of the pressure-by-volume diagram $(\mathrm{PxV})$, are often used to address and solve problems.

On the other hand, in potential thermodynamics, the description of thermodynamic systems through functions emphasizes the analytical aspect of this approach, whose explanatory power is based on the mathematical properties of the energy function, which foster alternative and equivalent representations of the system through the Legendre transformations [13, chap. 5] and where the integrability of the linear forms of multiple potentials reproduces the so-called Maxwell relations.

A apparent gulf seems to separate the cycle and potential versions of thermodynamics. However, the potential approach is heuristically dependent on the cycle approach, at least in Gibbs' formulation. By heuristically dependent, we mean more than a conceptual dependence, in the sense that the inherent concepts of thermodynamics were initially proposed and subsequently made clear through the representation by cycles, as is the case with the concepts of equilibrium, reversibility, absolute tem-

\footnotetext{
${ }^{4}$ More details on Carnot's cycle can be found in 24 .

${ }^{5}$ The notation $d^{\prime}$ is used here to emphasize that $\mathrm{Q}$ and $\mathrm{W}$ are not state functions, therefore, in a mathematical language $d^{\prime}$ is not an exact differential.
}

perature, energy, and entropy. The heuristic dependence is manifested in the fact that potential thermodynamics is formulated based on an analytical understanding of the diagrammatic representation peculiar to the thermodynamics of cycles. This understanding is analogous to that of Descartes's, which led him to establish a simple and direct line of communication (translation rules) between Euclidean geometry and algebra. In thermodynamics, this path is established by Gibbs and visible in the first two articles he wrote on the subject.

There are three articles in which Gibbs builds his version of thermodynamics: in the first two, the goal was to find the most appropriate way to represent thermodynamic systems possessing the peculiarity of being macroscopic and completely described by two independent variables, chosen from a set of five. In the first of these articles, "Graphical Methods in the Thermodynamics of Fluids" (April-May 1873), Gibbs analyzes and extends the properties of the diagrammatic representation, a fundamental tool in the thermodynamics of the time. The starting point is the usual $\mathrm{PxV}$ diagram. Looking at this through the first law, i.e., $d U=d^{\prime} Q-d^{\prime} W$, with $d^{\prime} Q=T d S$, for reversible processes, and $d^{\prime} W=P d V$, the variables $\mathrm{V},-P$ and $-W$ may be replaced respectively by $\mathrm{S}, \mathrm{T}$ and $\mathrm{Q}$ without the equations changing as a whole. The first remains unchanged, while the second is transformed into the third and the third into the second. This symmetry suggests that this diagram can be transformed into a temperature by entropy diagram, without changing its physical and geometrical properties, namely, the amount of heat used is equal to the mechanical work performed, the latter being determined by the area circumscribed by the cycle [26, p.9]. This reasoning by symmetry allowed him to introduce the TxS diagram as a tool for the analysis of thermodynamic processes, which implies simplifications for the study of phenomena, such as the one represented by the sequence of processes performed by the Carnot machine.

Greater attention, however, is dedicated to another diagram, $\mathrm{SxV}$, whose $\mathrm{x}$-axis represents the entropy values and the $y$-axis the volume values of great relevance in the representation of processes in which two or three phases of a substance coexist. In such cases, all processes are such that temperature and pressure remain constant, as a condition of the coexistence of different phases, with only the entropy and the volume of the parts changing. In a $\mathrm{PxV}$ graph, these processes are represented by a segment of a straight horizontal line, while in a SxT graph they are represented by a segment of a straight vertical line. This is the advantage of this diagram: it is more convenient to study the properties of substances, while the other two are more suitable to determine the work or the heat released in certain cases. The reason for the greater convenience of the $\mathrm{SxV}$ diagram for the study of phase changes is that the two variables chosen, entropy and volume, are extensive and not intensive, i.e., they are both proportional to the amount of substance, 
while pressure and temperature are not. The quantity of the substance in each state, therefore, is detected and expressed by the diagram [26, p.xv]. There is, however, a price to pay for the availability of more information. With a little bit of mathematics, it is possible to make explicit that such diagrams have a work scale factor that is given by $-\frac{d^{2} U}{d V} d s$, which means that this is not provided directly by the area between the curve representing the thermodynamic process and the volume axis, unlike what happens in the $\mathrm{PxV}$ and $\mathrm{TxS}$ diagrams, which have a constant scale factor equal to one. When the processes constitute a cycle, work and heat, which in $\mathrm{PxV}$ and TxS were given by the circumscribed area due to the fact that the first law, here reduced to $d^{\prime} Q=d^{\prime} W$, acquires the form $-\left(\frac{d^{2} U}{d V d S} d V d S\right)$. With the aid of the identities $P=-\frac{d U}{d V}$ and $T=\frac{d U}{d S}$, which Gibbs already knew, the scaling factor is also written $\frac{d P}{d S}$ and $-\frac{d T}{d V}$ [26, pp. 20-23].

As noted, the greater convenience of the $\mathrm{SxV}$ diagram in relation to the other two rests on the extensive nature of the volume and entropy values. In the case of a state change, as heat is exchanged with the environment, portions of the substance pass from one state to another, and those states coexist until the substance is completely converted. The extensive nature of these quantities allows for an exact tracking of the evolution of portions of the substance in each state through volume and entropy. In addition, representations through the mentioned quantities are equivalent to focusing attention on the substance and its characteristics, as transition curves and coexistence phases [26, p.24]. Just as in the thermodynamics of cycles, the diagram remains indispensable, but the cycles cease to be the method for the extraction of information from the physical system. This is directly available in the diagram, as in the case in which the region between the points where the substance is completely in the solid, liquid and gaseous state corresponds to a domain of volume and entropy values in which the three states coexist.

Interpreting this articles by Gibbs as the first step toward a theory of potential thermodynamics 13 , chap. $5]$, the knowledge of greatest relevance displayed here is not a new law or principle, but a way of representing thermodynamic systems, that includes the phase change phenomena. A historical fact confirms this judgment. In 1863, Thomas Andrews presented his experimental results about the coexistence conditions of solid, liquid and gaseous states and of the critical state, in some substances. According to Klein [27, pp.6-7], Andrews's discovery, which revealed an unexpected general property of matter, captured Gibbs' attention, especially since it addressed an issue not yet theoretically analyzed by the community. With his attention on this, Gibbs' first concern was to develop an adequate representation of substances in phase changes.

With this representation, processes can be described in terms of entropy and volume variations, but in this way and in accordance with the first law $(d U=T d S-P d V)$, the energy variation, another extensive quantity, can be seen as the combination of the variation of the other two extensive quantities. Gibbs's merit is that this view allows for an interpretation of the first law combined with the second law restricted to reversible processes $\left(d^{\prime} Q=\right.$ $d S / T)$ as an exact differential of an energy function $U=$ $U(S, V)$. Immediately, the $\mathrm{SxV}$ diagrams acquire a very precise meaning: they are the equivalent to projections of the process in the surface $U=U(S, V)$ on the plane. In other words, the empirical foundation contained in the first law, written as above, is also represented by a surface that represents the energy of the system, since the differential form is exact.

The diagrams representing the substance in two dimensions make place, therefore, for surfaces, which incorporate the former representation as projection planes. In this sense, they constitute a richer and more comprehensive representation than the two-dimensional diagram. This change in the representation space cannot be found in the work "Graphical Methods in the Thermodynamics of Fluids" (1873), although it is announced:

The possibility of treating the thermodynamics of fluids by such graphical methods as have been described evidently arises from the fact that the state of the body considered, like the position of a point in a plane, is capable of two and only two independent variations. It is, perhaps, worthy of notice, that when the diagram is only used to demonstrate or illustrate general theorems, it is not necessary, although it may be convenient, to assume any particular method of forming the diagram; it is enough to suppose the different stages of the body to be represented continuously by points upon a sheet. [26, p.32].

Change is the subject of Gibbs' second article, "A Method of Geometrical Representation of the Thermodynamic Properties of Substances by Means of Surfaces", exemplified by the fact that the

[...] properties of a fluid are determined by the relations which exist between the volume, pressure, temperature, energy, and entropy of a given mass of the fluid in a state of thermodynamic equilibrium [...] But all the relations existing between these five quantities for any substance may be deduced from the single relation existing for that substance between the volume, energy, and entropy [28, p.33].

This relationship consists in the differential expression of the first law $d U=T d S-P d V$, with the coefficients $T=\left(\frac{\partial U}{\partial S}\right)_{V}$ and $P=-\left(\frac{\partial U}{\partial V}\right)_{S}$, which is equivalent to interpreting energy as a function of entropy and volume, i.e., as a surface in the configuration space of rectangular coordinates energy, entropy and volume. Arranging V, $\mathrm{W}$, $\mathrm{U}$ respectively as the $\mathrm{X}, \mathrm{Y}, \mathrm{Z}$ axes, the temperature 
given by the energy derivative in relation to entropy, while the volume remains constant $\left(T=\left(\frac{\partial U}{\partial S}\right)_{V}\right)$ corresponds to the slope or tangent of the surface $\mathrm{U}(\mathrm{S}, \mathrm{V})$ in the direction $\mathrm{S}$ at each point or state of the fluid, while the pressure corresponds to the tangent of the surface in the direction $\mathrm{V}$, also for each point. This means that the tangent plane at each point of the surface is defined by a temperature and pressure value. In this sense, if one knows the surface $\mathrm{U}(\mathrm{S}, \mathrm{V})$, the other properties of the substance are obtained by derivation, namely, temperature and pressure, which are the state equations of the system. This is different than how James Thomson proceeded when he represented substances by the surfaces $\mathrm{T}(\mathrm{P}, \mathrm{V})$, from which, however, the state equations of the system cannot be directly inferred, by derivation. The conclusion is categorical:

It is evident, however, that the relationship between the volume, pressure and temperature affords a less complete knowledge of the properties of the body than the relationship between volume, entropy, and energy. 28, p.34].

The yellow plane (Figure 1) is tangent to the surface $\mathrm{U}(\mathrm{S}, \mathrm{V})$ at the point $\mathrm{P}$. With the state equations of temperature and pressure, given in the previous paragraph, these quantities resemble the vectors generating the tangent plane, therefore, directly derived from the expression of energy. On the other hand, when the analytical form of the energy is not yet known, with the values of the temperature and pressure pair obtained by the experiment, planes can be overlapped in such a way as to form the envelope of the surface $\mathrm{U}(\mathrm{S}, \mathrm{V})$. This is the idea behind the Gibbs-Duhem relationship: from the empirical basis represented by the values of temperature and pressure, we infer the expression of energy. 29, p.47]

Consequently, the representation of an object has an influence on its understanding. In one of these, more information needs to be added to the representation, in

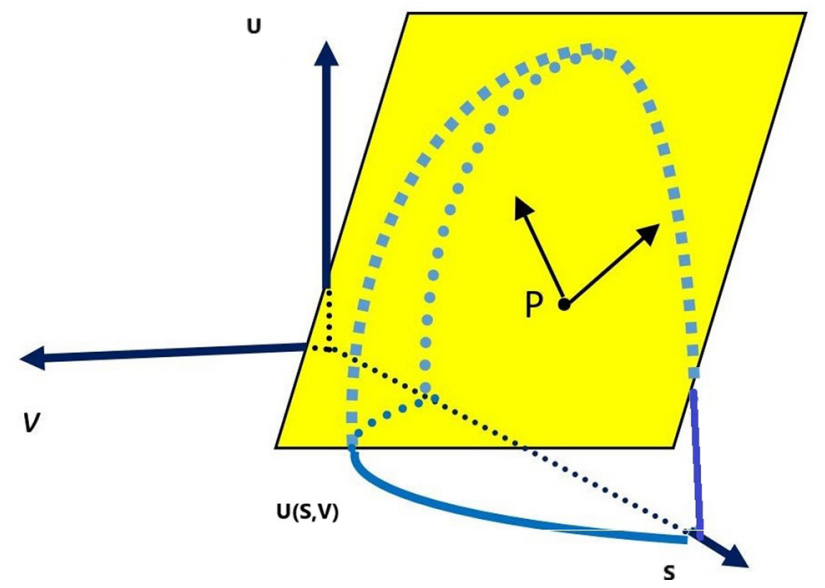

Figure 1: Gibbs' diagrammatic representation the form of additional equations, so that the five properties of the system can be determined. In the other, the representation contains all the information pertaining to the properties, with two of these being derived directly from the algebraic expression of the surface. The simplification operated by Gibbs is based on the compression and the logical order with which the extensive $(\mathrm{V}, \mathrm{S}, \mathrm{U})$ and intensive $(\mathrm{T}, \mathrm{P})$ properties are arranged. It is this logical relationship that provides more completeness to the representation using the volume, entropy and energy coordinates.

This mode of representation can also be applied to cases in which different portions of a body are in different states, always assuming that the whole is in thermodynamic equilibrium. At this point, however, it is necessary to introduce a distinction: the thermodynamic surface must be divided in two parts, one of which represents the homogenous states, called primitive surface, and the other the heterogeneous states, called derived surface. The previous designation follows from the fact that, given the first surface, the second one, the derivative, is immediately formed. The derived surfaces are constructed by tangent planes, and the values of the extensive quantities of the parts are summed together to compose the values of the whole, while the values of the intensive quantities are simply shared. Thus, the whole has the same temperature and pressure values as in equilibrium. The state, for example, in which the body is partly solid, partly liquid and partly vapor, is represented by a triangular plane on the primitive surface, which, as previously stated, represents only homogeneous states. As such,

[...] The position of the point determined by the volume, entropy, and energy of such a compound will be that of the center of gravity of masses proportioned to the masses of solid, liquid, and vapor placed at the three points of the primitive surface which represent respectively the states of complete solidity, complete liquidity, and complete vaporization, each at the temperature and pressure of the compound [26, p.36].

Since the temperature and pressure pair defines the plane tangent to a point on the primitive surface, the three points on the primitive surface and the compound states determined by the center of gravity belong to a common tangent plane. The points mentioned, which represent homogeneous states, constitute the vertices of a triangle that corresponds to the derived surface for the substance composed of three physical states. The points on the derived triangle all have identical temperature and pressure, and the extensive properties are determined there by the weighted average of the vertex values of each one, relative to the partial masses in each physical state: $V_{\text {Total }}=\left(\frac{M_{\text {sol }} V_{\text {sol }}+M_{\text {liq. }} V_{\text {liq }}+M_{\text {vap }} V_{\text {vap }}}{M_{\text {sol }} M_{\text {liq }} M_{\text {vap }}}\right)$, where $V_{\text {sol }}$, $V_{l i q}$ and $V_{v a p}$ are the values of the volume in the vertices referring to the states of complete solidity, complete 
liquidity and complete vaporization. The same is valid for entropy and energy.

As Gibbs himself pointed out, the $\mathrm{SxV}$ diagram is more appropriate to represent phase change phenomena, but the arrangement of the isobaric isotherms on it was not clear, a flaw that can be overcome through the representation by primitive and derivative surfaces. In this sense, the intellectual path taken by Gibbs to get to the thermodynamics of potentials begins with the appreciation of the cycles and its geometric support, which is the diagrammatic representation of thermodynamic phenomena. However, Gibbs expands the possibilities of representation, highlighting the potentialities of the $\mathrm{PxV}, \mathrm{SxT}$ and SxV graphs. The latter, as previously mentioned, is the most convenient for the representation of phase change phenomena, with no more need to resort to cycles. Of these, only the representative diagrammatic substrate remains. Next, Gibbs develops the idea, which is latent in the graph method, of the representation of the energy of surface substances, as emphasized above.

With surface representation, Gibbs is also able to characterize the critical points mathematically in a formal and broad way compared to the more intuitive way that came from the analysis of the isotherms in the vicinity of the critical point in the Andrews diagram, thus providing a theory for this new phenomena [26, p. 46]. This characterization is made through the resources that the geometric representation itself offers, such as the notion of normal curvature, contact order and tangent planes [26, p. 44-46]. A similar reading of Gibbs' second article will be improved in a paper still to be published, which aims, among other things, to detail how geometry was used to equate this problem mathematically and how this representative element resulted in a greater heuristic power of the Gibbs theory in comparison with that in force before him. It is precisely this aspect that leads us to understand Gibbs' thermodynamics, both in geometric and algebraic representation, as something more than a mere systematization of the thermodynamics before him.

There are physical reasons, implicit in this work by Gibbs that authorize us to make additional statements about $U=U(S, V)$. The first of these is linked to the fact that the temperature is measured on a positively defined absolute scale. Since $T=\left(\frac{\partial U}{\partial S}\right)_{V}$ is the slope of the tangent line to the curve described by energy in relation to entropy, at constant volume, it can be said that, since $T>0, \mathrm{U}(\mathrm{S}, \mathrm{V})$ as a growing monotonic function of S. Moreover, the principle of minimum energy is equivalent to stating that energy is a convex function of the entropy, its convexity being directly connected to the line tangent to the curve described by the function. The second assertion is related to the fact that the specific heat at constant volume $\left(C_{v}\right)$ - expressed by $\left(\frac{\partial^{2} U}{\partial S^{2}}\right)_{V}=$ $\left(\frac{\partial T}{\partial S}\right)_{V}=\frac{T}{C_{V}}$ - is always positive, in agreement with the condition of convexity of the function, which implies $\left(\frac{\partial^{2} U}{\partial S^{2}}\right)_{V} \geq 0$.
The notion of convexity used by Gibbs, especially in his second paper, is more intuitive and comes from Archimedean static, corresponding to the generalization that if a plane figure lies on one side of a line, its centroid lies on the same side of the line. Gibbs would have carried this notion to surfaces, but not to identify regions of convexity. As a physicist, his goals go beyond mathematics or pure geometry. He seeks to characterize the states of a substance in thermodynamic equilibrium as stable, metastable, neutral and unstable. And because of the strong intuitive connection between these two notions, in Physics, it is easy to read convexity where Gibbs is strictly speaking of stability. In fact, it may be that he understands them as synonyms. If he did it, we do not know, but we know that a contemporary of him did it.

Almost ten years after the publication of Gibbs's second paper, Paul Saurel, investigating the critical states of a binary system, finds that conditions similar to those mentioned in two paragraphs above, but which combine energy with the masses of the subsystems, are verified, declaring then that "thus at every point which corresponds to a phase in stable equilibrium the G (Gibbs potential) curve is convex toward the $\mathrm{m}$ (mass) axis" 30 . p.630]. The reason for this ambiguity is the absence of a rigorous and comprehensive formalization of convexity. As Wighman draws attention, like Saurel, Gibbs was also ambiguous in using these notions. This, however, was not for less, since a mathematical theorem combining convex functions with their convex conjugates, essential for the description of the system by different potentials, appeared only in 1948 [31, p. xxix]. Therefore, it is not surprising that the condition of convexity applied to energy, as found in Wighman, namely

$$
\begin{aligned}
& U\left[a S_{1}+(1-a) S_{2}, a V_{1}+(1-a) V_{2}\right] \leq a U\left(S_{1}, V_{1}\right) \\
& +(1-a) U\left(S_{2}, V_{2}\right)
\end{aligned}
$$

onde $S_{0} \leq S_{1}, S_{2}<\infty$ e $0 \leq a \leq 1$, is not present in the works of Gibbs [31, p.xi].

There is an equivalent definition of convexity, which requires the differentiability of the function. This definition can be applied to energy according to the version of Gibbs' second paper [32, p. 71], because there the differentiability of energy in relation to entropy and volume is assumed [26, p.33]. With this, the geometric property of the convexity is again recognized in the energy function.

In his next article, "On the equilibrium of heterogeneous substances", this geometric property is still implicit in the enunciation of the criterion of equilibrium and stability, which is the principle from which all other information about the system can be synthetically derived. Assuming the convexity of surface, energy is subject to Legendre's transformations, from which the other thermodynamic potentials are obtained. Therefore, ascending to the convexity of energy not only allows us to reach the (variational) principle of the theory, which is the 
criterion of equilibrium and stability, but also to establish that this is also valid for other potentials, which are no longer described in terms of independent variables (volume and entropy), but as pairs, such as volumetemperature, pressure-entropy and pressure-temperature.

That Gibbs's intention was to formulate a thermodynamics based on a principle of equilibrium and stability $(\partial U \geq 0$ or $\partial S \leq 0)$ is clear in a brief article summarizing the main points of "On the equilibrium of heterogeneous substances". There he recognizes that his inspiration was the Static, conceiving the potentials as a kind of forcefunction of the system [33, p. 441-442]. But to achieve the principles, he had to discover them by means of an auxiliary construction, the representation of the empirical basis by surface in $\mathrm{UxSxV}$ configuration space, which simulates Descartes's method of analysis.

The representation by surfaces is the heuristic instrument that favors the transition from the formalism of cycles to the formalism of potentials in thermodynamics, which is not presented explicitly in Gibbs' most famous work, "On the Equilibrium of Heterogeneous Substances", whose first part came out in 1875. In this work, surfaces are presented algebraically by the functions $U$ for energy or $\mathrm{S}$ for entropy the graphical element no longer appears, that are not just any function, but subject to the conditions that Gibbs calls equilibrium and stability criteria, which are variational principles. The criterion referring to energy assumes that the $U$ function (convex) is differentiable and increasing in relation to the entropy (concave function). However, such assumptions also ensure that the $\mathrm{U}$ function is subject to the Legendre transformations, which enable the information related to the energy of the system to be written in other ways, all of them equivalent. These are the other thermodynamic potentials, Helmholtz free energy, enthalpy, and Gibbs free energy. The integrability of the differentials of these potentials gives rise to the so-called Maxwell relations 29, pp.55-65 and 85-92].

The thermodynamics of potentials adapts and generalizes the phenomenology of phase transitions to chemical reactions, in which the homogeneous compounds of a heterogeneous substance react, forming new compounds. The idea is the same as changing physical states. The substance is composed of homogeneous components. The reaction between them causes portions of one to disappear and others to emerge. Thus energy is no longer a function of only two variables, as in the change of state of homogeneous substances. If there are two reactive substances, the energy of the system is a function of the type $U=U\left(S, V, m_{1}, m_{2}\right)$ - where $m_{1}$ and $m_{2}$ are the masses of the reactants - which is a hypersurface in a space with five dimensions. The visualization is obviously impractical, which explains, in part, the difficulty of perceiving the geometric aspect, now applied to hypersurfaces, as the heuristic model of the thermochemistry of potentials
6 Therefore, the thermodynamics of potentials, in addition to being more logically coherent than Clausius's thermodynamics, is also more prosperous, since in its formalism it contains the new discipline of thermochemistry. Such prosperity is witnessed by Maxwell when he states that "Professor Gibbs has made a most important contribution to science by giving us a mathematical expression for the stability of any given phase (A) of matter with respect to any other phase (B)" 35, p.821]

Descartes's method of discovery illuminates the intellectual itinerary of the construction of the thermodynamics of potentials. We are not saying that Gibbs drew directly from Descartes; the influence is indirect, but it is an extraordinary example of the application of the analytical method. In fact, Gibbs is concerned first of all with adequately representing the processes during through which different states of matter coexist. This is the problem for which he seeks a solution, in addition to that of the critical points discovered by Thomas Andrews. This last aspect was studied elsewhere [36, p.119-121].

That is why he explores the properties of the $\mathrm{SxV}$ diagram - which as a heuristic resource is similar to that employed by scientists until Clausius. The diagrammatic representation $\mathrm{P} \times \mathrm{V}$ operates the calculation of the mechanical work performed during a thermodynamic process through the area under the curve; in the $\mathrm{S} \times \mathrm{V}$ diagram, this calculation is difficult because the scale factor is no longer the unit. There does not seem to be any advantages in adopting it.

Here, then, comes into play the genius of Gibbs, who identifies an intermediate idea, playing a role similar to that played in the examples of Descartes. The $\mathrm{SxV}$ diagram associated with the first law - restricted to reversible processes - suggests that if the differential $d U=T d S-P d V$ is exact, the one described in the diagram is interpreted as a projection of processes (lines) that occur on a surface $\mathrm{U}(\mathrm{S}, \mathrm{V})$. The intermediate idea is precisely that the differential form is exact, that it be integrable. However, diagrammatic (two-dimensional) representation becomes a geometric (three-dimensional) representation with the aggregation of elements that make it more prosperous as a language and, therefore, give it more significant heuristic power. In fact, while in the diagrammatic representation the problems are approached with the help of the properties of the curves on the graph and the area under the curve (mechanical work) in the geometric representation, tangent planes, tangent lines, concavity, curvature and contact order are used besides the properties of the curves on the surface to characterize, translate and solve the problems of heat science. The possibilities are much more significant.

This is a new formalism that can not be reduced to a mere systematization, which is commonly understood as an act of organizing something according to a system

${ }^{6}$ More details on the thermodynamics of cycles and potentials can be found in 23], which, as a thesis on the history and philosophy of thermodynamics, is a further development of 34 . 
or reason. In the present case, it would be a matter of disposing general laws and particular conditions in order to proceed with deductive or inductive arguments 37 , p. 173-7]. If so, Gibbs would frame the problems in terms of the two fundamental laws and other conditions. But he does not even name them for that purpose. As we have observed, he does so with a geometric language, absent in classical formulation. This is the point. What Gibbs discovered was that thermodynamics could be put into a geometric language, and this discovery was made according to a method similar to Descartes' method of analysis.

To sum up, Gibbs' work begins with the appreciation of the elements representing the thermodynamics of cycles, the diagrams. This perception already seems to us to be valuable in itself, since - starting especially with William Thomson and Clausius - the diagrams are shifted to the periphery of theory, as auxiliary or even ad hoc hypotheses. At the core are the two fundamental laws, isolated and independent of any representative aspect. However, the problems that Gibbs had before his eyes demanded more of the thermodynamic magnitudes, especially energy. The principle of energy conservation, although fundamental to the development of science, provides a trivial geometric intuition, compared to what occurs in the thermodynamics of potentials. This first step by Gibbs reveals his intention to bring a representative element back to the nucleus of the theory.

To describe the processes of change and coexistence of different phases, Gibbs uses the $\mathrm{SxV}$ diagram. This diagram, which supports a vast set of thermodynamic phenomena with zero energy variation - TdS $=\mathrm{PdV}$ forms the basis of Gibbs' system of knowledge, similar to the dynamics of shocks in the problem of the nature of light, for Descartes. For him, the base was associated with the intermediate idea of the model of light, which provided the characterization of light as an oscillating matter whose velocity increases in the passage to denser means. From this idea he derived the law of refraction. For Gibbs, the intermediate idea associated with the base is the first law - restricted to reversible processes - leading to a geometric thermodynamics capable of solving the problem of representing the transition of states of matter and the characterization of critical points. Therefore, the method of analysis is the instrument to discover or construct solutions.

One consequence of the application of Descartes' scientific method is the expansion of the knowledge base. Once a proposition is justified, it becomes part of that basis. In the present case, the accuracy of the linear form adds to energy the geometric properties inherent to the surface $\mathrm{U}=\mathrm{U}(\mathrm{S}, \mathrm{V})$. Therefore, it should be noted that the geometrization of energy occurs through the intervention of the intermediate idea which extends the knowledge basis from diagrammatic thermodynamics, to geometric thermodynamics.
However, there is an essential difference between the two versions as regards the representative element. In the first version, the physical quantities involved, including energy and entropy, are extrinsically diagrammatic. Their relative values are collected empirically and then graphically implemented. In the second version, they are intrinsically geometric.

It is true that their values can be obtained by experiment and that the surface $U=U-(S, V)$ is subsequently formed as in the previous version, but energy and entropy have the property of being convex and concave, respectively, as we have argued before. It is a fusion of physics and geometry, not a mere juxtaposition.

This fusion between physics and geometry is what authorizes the variational formulation of principles related to energy and entropy. These principles are not formally present in Gibbs's second article, only in the third - "On the Equilibrium of Heterogeneous Substances"(1878).

When using the geometric formulation to solve the problem of homogeneous substances in equilibrium, the problem of heterogeneous substances immediately arose, requiring the incorporation of the masses of the different components. For example, for a heterogeneous substance, composed of two homogeneous ones, the differential equivalent to the first law is written $d U=T d S-P d V+$ $\mu_{1} d m_{1}+\mu_{2} d m_{2}$. The indices 1 and 2 refer to each of the homogeneous masses.

As in "A Method of Geometrical Representation of the Thermodynamic Properties of Substances by Means of Surfaces", the solution is also constructed following the method of analysis, but the basis is now the thermodynamics of the homogeneous substances. The intermediate idea is to identify the differential referring to the first law as an exact linear form, which leads to the interpretation of energy as a hypersurface $U=U\left(S, V, m_{1}, m_{2}\right)$ and to the recognition of $\mu=\left(\frac{\partial U}{\partial m}\right)_{S, V}$, as the chemical potential of each homogeneous substance. In this context, the geometric language (convexity and tangent planes) is no longer very useful, since the visualization of these structures in hyperspace is not feasible. The best thing now is to translate these properties into algebraic language.

This is why the principle of equilibrium and stability appears explicitly in "On the Equilibrium of Heterogeneous Substances" (1878), reflecting the convex nature of energy and entropy and other thermodynamic potentials (Helmholtz potential, enthalpy, and Gibbs potential). They mirror the enveloping of the information contained in the energy function by tangent hyperlines or tangent hyperplanes by the implicit use of the Legendre transformation.

The mentioned potentials are also subject to the same variational principle. The hypergeometric character of the thermodynamics of heterogeneous substances requires that it be represented algebraically. The solutions implemented by Gibbs go through the appreciation of the representative aspect. At first, the valorization of the diagrams leads to a geometrically represented thermody- 
namics, which later, has to be amplified so that it can be generalized to heterogeneous substances. This bring about the thermodynamics of potentials, algebraically represented.

The way one moves from one representation to the other - which is equivalent to solving the problem proposed in each case - is through the analytic method. Here it is not the geometry or the algebra of the theory, but that which leads to geometrization and algebrization from a knowledge base, as already mentioned. Gibbs operates with Descartes' method of analysis in this way, solving problems by constructing representations with these capabilities. In this sense, it can be said that one representation has more heuristic power than another, insofar as its language is more productive and adapted to explain more natural phenomena. In the case of heterogeneous substances, the new algebraic language is capable of encompassing phenomena hitherto outside the semantic reach of thermodynamics, thus giving rise to a new field of knowledge: thermochemistry.

We believe that we have performed a rational reconstruction of Gibbs's findings through Descartes' method of analysis. These discoveries are identified with the solution of problems, placed on a lower (representative) level than that of the solution, in the ladder that goes from the diagrammatic representation to the algebraic, through the geometric one. Success in this endeavor consists in the recognition that the representative element forms an integral part of these solutions, making them viable. On the other hand, we agree that the thermodynamics of potentials can be understood as a rational reconstruction of classical thermodynamics, in Carnap's terms, for whom:

By rational reconstruction is meant here the searching of new definitions for old concepts. The old concepts did not ordinarily originate by way of deliberate formulation but in more or less unreflected and spontaneous development. The new definitions should be superior to the old in clarity and exactness, and, above all, should fit into a systematic structure of concepts 38, p.v].

In this perspective, the concepts of energy and entropy - defined in the contexts of the first and second laws - would pass to the context of the principle of equilibrium and stability, acquiring, with this contextual transfer, the multi-representation by other potential concepts, the connaturality with the phase transitions, the characterization of critical points and the description of heterogeneous substances. The gains with the new definitions would not be so much in clarity and accuracy, but in strengthening the logical cohesion of the systematic structure of concepts.

It is faced with this panorama opened by Lagrange's analytical mechanics, extended to optics by Hamilton and to thermodynamics by Gibbs, that Duhem proposes a rational and empirically testable method for physics, which surpasses the mechanistic method. In this context, Duhem advocates the unification of physics based on an abstract method, which is the proper method of potential thermodynamics, where empirical laws are deduced from a potential function subject to a variational principle. In the opposite direction, the potentials - that is, the principles of the theory - can be obtained from the empirical basis represented by the state equations with the aid of the Gibbs-Duhem equation, by integration. The capacity to ascend in this way to the principles of manifest theory, at the same time as the are logically bound to the empirical foundation, and the control of theory by experience as a result of this bond - others would appeal to the idea of falsification - an intrinsic feature of scientific knowledge [13, chap. 5], [10, p. 215].

\section{Conclusions}

The construction of the thermodynamics of potentials was accomplished by Gibbs through a process in which the representation of the energy of a homogeneous substance in the most adequate configuration space, that of $U \times S \times V$, is followed, for physical and mathematical reasons, by the enunciation of the equilibrium and stability criterion, the highest principle and the conclusion of the analytical movement. In this context, the ascent to the aforementioned principle would be greatly hampered were it not for the adequate representation of the thermodynamic systems, which incorporated at the same time a sufficiently versatile operability: geometric representation. Thus, Gibbs's first article, the one which seemed less interesting because it brought less novelties and no predictions, acquires a special relevance. It is as if Gibbs were looking for the best way to represent phase transition phenomena before solving the problem of constructing a thermodynamics that incorporated them. And in finding it, the solution becomes intuitive.

Descartes's method of analysis may not consist in a method of logical justification, but it presents itself as an authentic method of discovery in which the invention or construction of an adequate representation of the proposed problem - within which a specific method operates - with an intermediate idea allows us to ascend to the highest principle, the starting point for logical deductions. Even though analysis does not possess the logical force of synthesis, according to the Cartesian model it authorizes us to speak of rational reconstruction in science. We believe that we have presented such a reconstruction in the case of Gibbs' thermodynamics, while illustrating some of the reasons that led Duhem to see in thermodynamics, according to this formalism, the center around which physics should unify, replacing mechanicism . 


\section{Acknowledgments}

The authors are grateful to the Institute of Physics of the University of Brasilia, the Departments of Philosophy and of Economics of the University of São Paulo for their support. We are also grateful to the referees whose observations and suggestions helped to improve this work.

\section{References}

[1] K. Popper, Objective Knoledge: An Evolutionary Approach (Oxford University Press, Oxford, 1979).

[2] H. Reichenbach, Experience and predictions: an analysis of the foundations and the structure of knowledge (The University of Chicago Press, Chicago 1961).

[3] K. Popper, The Logic of Scientific Discovery (Routledge Publishing, New York, 1999).

[4] C.G. Hempel, Philosophy of natural science (PrenticeHall, Princeton, 1966).

[5] H. Feigh, in Analyses of Theories and Methods of Physics and Psychology, edited by M. Radner and S. Winokur (University of Minnesota Press, Minneapolis, 1970), p. 03.

[6] W.C. Salmon, in Historical and Philosophical Perspectives of Science, edited by R.H. Stuewer (University of Minnesota Press, Minneapolis, 1970), p. 68.

[7] P. Hoyningen-Huene, in Revisiting discovery and justification - Historical and philosophical perspectives on the context distinction, edited by J. Schickore and F. Steinle (Springer, Netherlands, 2006), p. 119.

[8] F.A. Kekulé, Bulletin de la Société Chimique de Paris, 3, 98 (1865)

[9] W. Whewell, Novum Organum Renovatum (John W. Parker and Son, West Strand, 1858).

[10] J.R.N. Chiappin, Ciência e Filosofia 5, 155 (1996).

[11] R. Descartes, Discours de la Méthode et Essais de cette Méthode: La Dioptrique, Les Méteores, La Géometrie (Librairie Philosophique J. VRIN, Paris, 1996).

[12] S. Neuvius, René Descartes' foundations of analytic geometry and classification of curves, Thesis Basic Level, Uppsala University, Uppsala (2013).

[13] J.R.N. Chiappin, Duhem's Theory of Science: The Interplay Between Philosophy and History of Science, PhD Dissertation, University of Pittsburgh, Pittsburgh (1989).

[14] R. Descartes, Les Méditations métaphysiques de René Descartes touchant la première philosophie (H. Leoras, Paris, 1661).

[15] Z. Loparic, Descartes Heurístico (UNICAMP, Campinas, 1997).

[16] C.A. Battist, O método de análise de Descartes: da resolução de problemas à constituição do sistema do conhecimento (Edunioeste, Cascavel, 2002).

[17] J. Hintikka and U. Remes, The method of analysis: its geometrical origin and its general significance (D. Reidel Publishing Company, Dordrecht, 1974).

[18] J.R.N. Chiappin, Discurso 43, 253 (2013).

[19] J.R.N. Chiappin and A.C. Leister, Discurso 39, 107 (2009).

[20] P. Fermat, Traduction des Écrits Latins de Fermat, in Oeuvres de Fermat edited by P. Tannery and C. Henry (Gauthier-Villars et Fils, Paris, 1996), v. 3, p. 149
[21] C.C. Laranjeiras, J. Lucena and J.R.N. Chiappin, Rev. Bras. Ens. Fis. 39, e4602 (2017).

[22] J.L. Lagrange, Mécanique Analytique (Jacques Gabay, Paris, 1989).

[23] J.L. Silva Abordagens Comparativas de Ciclos e de Potenciais da Termodinämica: Escolha Racional ou Pragmática? PhD Dissertation, Universidade de São Paulo, São Carlos (2015).

[24] C.C. Laranjeiras and S.I.C. Portela, Physics Education 51, 055013 (2016).

[25] J. Lucena and J.R.N. Chiappin, Principia: an international journal of Epistemology 21, 291 (2017).

[26] J.W. Gibbs, The Collected Works of J. Willard Gibbs (Longmans, Green and Co., New York, 1931), V.I.

[27] M. J. Klein, in Proceedings of the Gibbs symposium (Yale University, New Haven, 1989).

[28] J.W. Gibbs, The Collected Works of J. Willard Gibbs (Longmans, Green and Co., New York, 1931), V.II.

[29] S.R.A. Salinas, Introduction statistical physics (Springer, New York, 2001).

[30] P. Saurel, J. Phys. Chem., 6, 629 (1902).

[31] A.S. Wighman, in Convexity in the theory of lattices gases, edited by R.B. Israel (Princeton University Press, Princeton, 1979).

[32] F. Wreszinski, Termodinâmica (Editora da Universidade de São Paulo, São Paulo, 2003).

[33] J.W. Gibbs, American Journal of Science and Arts XVI, (1978).

[34] C.C. Laranjeiras O Programa de Pesquisa de Ludwig Boltzmann para a Mecânica Estatística. PhD Dissertation, Universidade de São Paulo, São Paulo (2002).

[35] J.C. Maxwell, The London, Edinburgh, and Dublin Philosophical Magazine and Journal of Science, 16, 818 (1908).

[36] M.J. Oliveira, Equilibrium Thermodynamics (Springer, Berlin, 2013).

[37] C. G. Hempel, Aspects of Scientific Explanation and Other Essays in the Philosophy of Science (Free Press, New York, 1965).

[38] R. Carnap, The Logical Structure of the World and Pseudo Problems in Phylosophy (The Open Court, Chicago, 1969). 